\title{
Correspondence
}

\section{Trigeminal nerve blockade, following inadvertent dural puncture - was there a connection?}

\section{To the Editor:}

The insertion of an epidural for pain relief in labour was complicated by a dural puncture. During the subsequent infusion the patient complained of numbness over her face. There was no accompanying tinnitus or circumoral numbness and pulse and blood pressure remained within normal limits. On examination an area of numbness, to pain and cold, was confined to the bilateral distribution of the maxillary division of the fifth cranial nerve. The epidural infusion was stopped and the numbness regressed over the next $20 \mathrm{~min}$.

Trigeminal nerve block following epidural analgesia has been reported before ${ }^{1,2}$ but this patient did not have Horner's syndrome. In previous reports there was no associated dural puncture. One possible mechanism is excessive cephalad spread of the local anaesthetic eventually reaching the spinal tract and/or nucleus of the trigeminal nerve and the cervical sympathetic fibres accounting for the trigeminal nerve block and Horner's syndrome in previous reports.

It has been suggested that excessively high subarachnoid blocks can occur, when there has been a subsequent epidural injection or infusion, due to increased pressure in the epidural space that causes cephalad spread. ${ }^{3}$ Some local anaesthetic may have entered the subarachnoid space through the dural puncture site and with the concomitant epidural infusion caused a greater cephalad spread than would normally occur thus explaining the trigeminal sensory loss, it is unlikely that the catheter entered the subarachnoid space as there was no evidence of a subarachnoid block, other than hypotension, which can be explained by other mechanisms.

The occurrence of trigeminal nerve blockade in this patient may or may not be related to the previous dural puncture. Patients with epidural infusions may complain of odd facial symptoms. These may be due to high plasma concentrations of local anaesthetics or, alternatively, to excessive cephalad spread of local anaesthetic.

J. O'Hanlon BSc MB FFARCSI

I. Bali PhD FFARCSI

P. Leyden MB FFARCSI

Department of Anaesthetics

Royal Group of Hospitals

Grosvenor Road

Belfast, BT12 6BA

$\mathrm{N}$. Ireland

\section{REFERENCES}

1 Sprung J, Haddox DJ, Maitra-D'Cruze AM. Horner's syndrome and trigeminal nerve palsy following epidural anaesthesia for obstetrics. Can J Anaesth 1991; 38: 767-71.

2 Gazmuri RR, Ricke CA, Dagnino JA. Trigeminal nerve block as a complication of epidural anesthesia. Reg Anesth 1992; 17: 50-1.

3 Beck GN, Griffiths $A G$. Failed extradural anaesthesia for Caesarean section. Complications of subsequent spinal block. Anaesthesia 1992; 47: 690-2.

\section{Visual evaluation of TOF and DBS fade using a rubber band}

To the Editor:

After train-of-four (TOF) stimulation the thumb does not return to the baseline prior to the first three twitches. ${ }^{1,2}$ Recently Brull et al. ${ }^{1}$ have shown that the TOF ratio may be overestimated by visual evaluation because of baseline shift of the thumb. We hypothesized that if the thumb was kept abducted using a rubber band when TOF or $\mathrm{DBS}_{3,3}$ was delivered, the TOF ratio $\left(T_{4} / T_{1}\right)$ or $D_{3,3}$ ratio $\left(D_{2} / D_{1}\right)$ above which fades could no longer be detected visually would increase.

Thirty adult patients were studied. After administration of vecuronium $0.1 \mathrm{mg} \cdot \mathrm{kg}^{-1}$, TOF and $\mathrm{DBS}_{3,3}$ stimuli were applied. In the RB group $(n=15)$ the thumb of the investigated arm was kept abducted using a rubber band as a preload of $150 \mathrm{~g}$ (Figure) but in the control group $(n=15)$ the thumb was unrestrained.

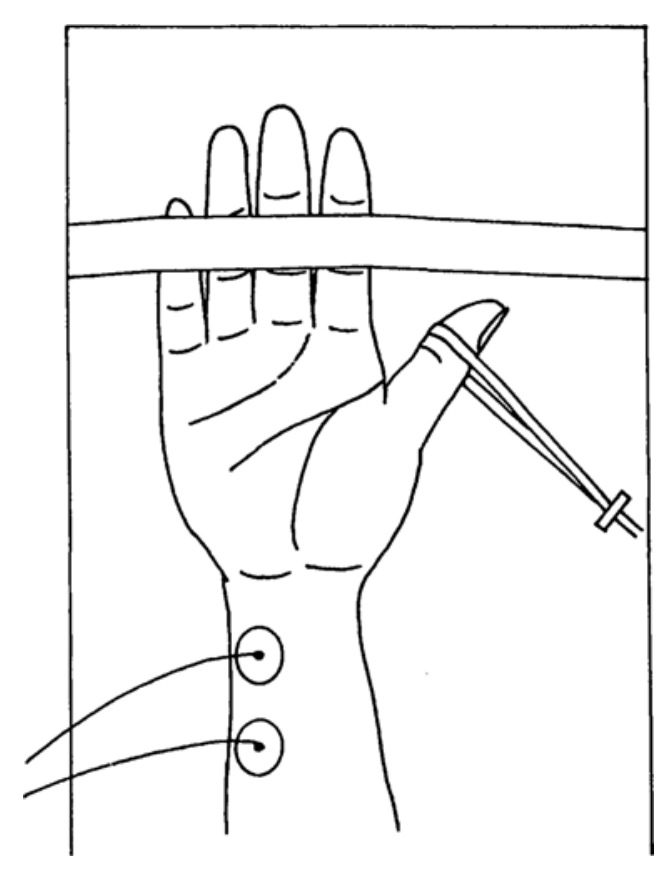

FIGURE 
The TOF ratio at visual disappearance of TOF fade in the $R B$ group was higher than that in control group $(0.515 \pm 0.061$ vs $0.401 \pm 0.084$, mean $\pm \mathrm{SD}, P<0.001)$. The $\mathrm{DBS}_{3,3}$ ratio at the visual disappearance of $\mathrm{DBS}_{3,3}$ fade in $\mathrm{RB}$ group was higher than in the control group $(0.74 \pm 0.05$ vs $0.631 \pm 0.05, P<$ 0.000001 ). These results suggest that by using a rubber band to keep the thumb abducted, the visual detection of TOF fade and $\mathrm{DBS}_{3,3}$ fade is easier.

Yuhji Saitoh MD

Kazuhiko Nishimura MD

Department of Anesthesiology and Critical Care Medicine

Faculty of Medicine

Tokyo Medical and Dental University

\section{REFERENCES}

1 Brull SJ, Silverman DG. Real time versus slow-motion train-of-four monitoring: a theory to explain the inaccuracy of visual assessment. Anesth Analg 1995; 80: 548-51.

2 Silverman DG, Connelly NR, O'Connor TZ, Garcia R, Brull SJ. Accelographic train-of-four at near-threshold currents. Anesthesiology 1992; 76: 34-8.

\section{La consultation préhospitalière d'anesthésie en Suisse francophone}

\section{A la rédaction:}

La consultation préhospitalière d'anesthésie (CPHA) ambulatoire est une pratique institutionnelle dans de nombreux pays tels la France, l'Autriche, les Etats-Unis ou le Canada. ${ }^{1-6} \mathrm{La}$ CPHA est avantageuse pour le patient qui bénéficiera d'un cadre relationnel favorable à la discussion et à l'information. L'anesthésiste peut organiser avant l'hospitalisation, une consultation spécialisée, initier ou optimaliser un traitement médical, définir avec le chirurgien la stratégie périopératoire. La CPHA permet finalement une réduction des coûts en évitant les examens paracliniques inutiles ou « de routine ", en diminuant le délai entre l'hospitalisation et l'opération et en améliorant le rendement du bloc opératoire, évitant les reports de dernière heure pour des patients insuffisamment préparés. ${ }^{3}$

En Suisse, la CPHA n'existe que de manière anecdotique, probablement lié au fait que le domaine de la santé a été touché plus tardivement par des restrictions budgétaires. Aussi, avant de présenter à nos autorités sanitaires un projet de CPHA, nous avons voulu connaître l'opinion d'anesthésistes, de chirurgiens et d'administrateurs hospitaliers.

Un questionnaire a été envoyé à 100 anesthésistes, 100 chirurgiens et 74 administrateurs, établis en Suisse francophone. Les réponses ont permis de sonder l'attitude des participants envers la CPHA. Soixante-quatre anesthésistes, 72 chirurgiens et 54 administrateurs ont répondu, dont la majorité travaillait dans une institution publique depuis plus de 10 ans. Les anesthésistes et les chirurgiens (97\% et $92 \%$ ) se sont déclarés plus intéressés à la CPHA que les administrateurs (79\%). A l'unanimité, les anesthésistes souhaitent la CPHA et
90\% la jugent nécessaire pour améliorer leurs prestations, alors que les chirurgiens, qui ont la même opinion, s'expriment de manière moins marquée (respectivement $79 \%$ et $73 \%$ ). Moins d'administrateurs sont favorables (60\%). Les anesthésistes $(78 \%)$ sont plus nombreux que les chirurgiens (50\%) à penser que le patient bénéficiera de la CPHA, que celle-ci peut diminuer le délai entre l'hospitalisation et l'opération (63\% d'anesthésistes, $40 \%$ de chirurgiens), ou qu'elle permet une meilleure gestion du bloc opératoire $(77 \%$ d'anesthésistes, $40 \%$ de chirurgiens). Les administrateurs ne se sont pas prononcés quant aux avantages pour le patient; mais $44 \%$ pensent que la CPHA peut réduire la durée de l'hospitalisation et $60 \%$ et améliorer la gestion du bloc opératoire. Le pourcentage d'anesthésistes (60\%) et de chirurgiens (53\%) estimant que l'organisation de la CPHA se heurtera à des problèmes administratifs est plus élevé que celui des administrateurs (29\%). Plus d'anesthésistes (55\%) et d'administrateurs $(40 \%)$ que de chirurgiens (24\%) estiment que les difficultés d'une CPHA sont liées à des obstacles financiers. Enfin, les administrateurs seuls pensent que la CPHA est du ressort de l'anesthésiste, alors que $13 \%$ des anesthésistes et $40 \%$ des chirurgiens suggèrent que la CPHA soit réalisée par d'autres spécialistes (interniste, cardiologue et généraliste).

Pour des raisons linguistiques, nous avons limité ce sondage à la Suisse francophone. Le nombre de réponses obtenues démontre l'intérêt des participants; il est toutefois surprenant que plus de chirurgiens que d'anesthésistes aient répondu, alors que ces derniers devraient se sentir les premiers concernés par la CPHA. Le pourcentage élevé des chirurgiens intéressés (92\%) est surprenant et probablement lié, comme suggéré récemment, au fait que la CPHA améliore la prise en charge globale des patients. ${ }^{4}$ L'amélioration des prestations anesthésiques, la rationalisation de l'hospitalisation et du bloc operatoire confirment l'efficacité de la CPHA. ${ }^{3}$ Elle permet de réduire le temps d'hospitalisation préopératoire ${ }^{5}$ et les délais et annulations d'une opération par le facteur dix. ${ }^{7}$ Les obstacles adminstratifs ou financiers soulevés plus souvent par les anesthésistes que par les autres participants sont le reflet de la situation en Suisse, où l'anesthésiste ne bénéficie par d'une infrastructure de consultation ni d'une tarification officielle. Contrairement à d'autres pays, ${ }^{3,4}$ le système de santé suisse ne recouvre pas la CPHA, ce qui pourrait expliquer que $13 \%$ des anesthésistes proposent de la déléguer à d'autres spécialistes. Pour conclure, notre sondage a monté que la CPHA était souhaitée et jugée nécessaire plus spécialement par les anesthésistes. Nos résultats devraient donc nous encourager à développer la CPHA dans notre pays, en exigeant qu'elle soit reconnue au même titre que les consultations d'autres spécialités médicales et que cette approche de l'anesthésie soit inclue dans les programmes de formation postdoctorale.

\section{Dr. C.E. Klopfenstein}

Dr. A. Foster

Département d'Anesthésiologie, Hôpital Cantonal

Universitaire, Genève, Suisse

\section{REFERENCES}

1 Lee A. The anesthetic out-patient clinic. Anaesthesia $1949 ; 4$ : 169-74.

2 Loder RE, Richardson HJ. Preoperative anaesthetic outpatient clinic. Lancet 1954; $2: 1177-8$.

3 Guilmet $C$. Vers une définition de la consultation d'anesthésiologie. Agressologie 1976; 17: 125-34. 